\title{
Endoscopic Radial Artery Harvest in Coronary Artery Bypass Surgery: Clinicopathological Evaluation
}

\author{
Amir Mohamed 1,2, Kamelia Velikova3 ${ }^{3}$ Ahmed Nabil'4, Amr Algarhy5, Mohamed Badawy, \\ Adel Maher 1,7, Ashraf Abdelsalam ${ }^{5,8}$, Jamal Al-Fadhli ${ }^{5}$, Riyad Tarazi', Nael Al-Sarraf ${ }^{5 *}$ \\ ${ }^{1}$ Department of Cardiac Surgery, AlDabbous Cardiac Center, Adan Hospital, Hadiya, Kuwait \\ ${ }^{2}$ Department of Cardiothoracic Surgery, Alexandria University, Alexandria, Egypt \\ ${ }^{3}$ Department of Histopathology, Adan Hospital, Hadiya, Kuwait \\ ${ }^{4}$ Department of Cardiology, Al Dabbous Cardiac Center, Adan Hospital, Hadiya, Kuwait \\ ${ }^{5}$ Department of Cardiac Surgery, Chest Diseases Hospital, Kuwait City, Kuwait \\ ${ }^{6}$ Department of Cardiothoracic Surgery, Ain Shams University, Cairo, Egypt \\ ${ }^{7}$ Department of Cardiothoracic Surgery, Aswan University, Aswan, Egypt \\ ${ }^{8}$ Department of Cardiothoracic Surgery, Zagazig University, Zagazig, Egypt \\ Email: *trinityq8@hotmail.com
}

How to cite this paper: Mohamed, A., Velikova, K., Nabil, A., Algarhy, A., Badawy, M., Maher, A., Abdelsalam, A., Al-Fadhli, J., Tarazi, R. and Al-Sarraf, N. (2019) Endoscopic Radial Artery Harvest in Coronary Artery Bypass Surgery: Clinicopathological Evaluation. World Journal of Cardiovascular Surgery, 9, 15-23. https://doi.org/10.4236/wics.2019.92002

Received: January 20, 2019

Accepted: February 12, 2019

Published: February 15, 2019

Copyright $\odot 2019$ by author(s) and Scientific Research Publishing Inc. This work is licensed under the Creative Commons Attribution International License (CC BY 4.0).

http://creativecommons.org/licenses/by/4.0/

(c) (i) Open Access

\begin{abstract}
Objectives: The radial artery is used as a second arterial conduit in coronary artery bypass surgery. However, concerns exist about the thermal injury incurred to the radial artery during endoscopic harvest. We sought to assess this effect both histologically and radiologically. Methods: From February 2015 to March 2016, 50 consecutive patients undergoing coronary artery bypass surgery utilizing endoscopically harvested radial artery were prospectively studied. Computerized tomography coronary angiography scan was performed randomly in 8 patients who also had their respective radial artery analyzed histologically at the time of harvest. Standard coronary angiography was also performed in 4 additional patients driven by symptoms recurrence. Results: All patients were available for follow up at 1 year following the surgery. There was no mortality during follow-up. Three patients (6\%) developed transient complications in the harvest arm which resolved. All radial arteries were patent on follow-up imaging. Histological examination showed integrity of the arterial wall, intact endothelial lining, patent lumens and no significant pathological abnormalities. Conclusion: The use of endoscopic radial artery harvest was associated with few self-limiting morbidities and excellent patency rates. In addition, there was no evidence of any thermal or traumatic injuries sustained to the radial arteries when examined histologically. The use of endoscopically harvested radial arteries appears safe with excellent results.
\end{abstract}




\section{Keywords}

Radial Artery, Endoscopic Harvest, Coronary Artery Bypass, Histology

\section{Introduction}

The radial artery is increasingly used as a second arterial conduit in coronary artery bypass graft (CABG) surgery [1] [2]. This has been largely due to its excellent patency rates and adequate length with less morbidity associated with its harvest. The use of endoscopic radial artery harvest is gaining popularity in recent years owing to smaller incisions, greater patient satisfaction and less complications in the harvested arm while maintaining similar patency rates to the radial artery harvested by open technique [3] [4] [5]. There are still some concerns that by using endoscopic harvesting technique, the radial artery might be compromised by thermal injury which may lead to inferior long-term patency rates [6]. In this paper, we sought to assess the effect of endoscopic radial artery harvest on thermal injury incurred to the radial artery both histologically and radiologically. In addition, we report our results of endoscopic radial artery harvest both in the short and mid-term.

\section{Materials and Methods}

\subsection{Patients}

From $1^{\text {st }}$ February 2015 to $1^{\text {st }}$ March 2016, 50 consecutive patients above the age of 18 years old who underwent first time isolated CABG utilizing radial artery as a conduit were prospectively studied in the departments of cardiac surgery at Chest Diseases Hospital and Al Dabbous cardiac center in Kuwait. Inclusion criteria included age above 18 years old, life expectancy more than 2 years, absent contrast allergy, isolated CABG procedure, non-emergency CABG, absence of contraindications to radial artery harvest, presence of non-dominant left arm, normal kidney function, and suitable coronary anatomy. Radial artery was placed on the second-best coronary artery system with stenosis $>70 \%$ in the left coronary system or $>90 \%$ in a right coronary artery territory as per American heart association (AHA) guidelines. All demographics were recorded, and all patients were followed up clinically for one year after the surgery. Computerized tomography (CT) coronary angiography scan was performed randomly on 8 patients who also had their respective radial artery analyzed histologically at the time of harvest. Only 8 patients were histologically studied for two reasons: first we insisted on having $1 \mathrm{~cm}$ length of radial artery remaining after grafting to adequately study histologically and second reason was due to lack of funding. Standard coronary angiography was required in 4 additional patients based on recurrence of angina postoperatively. All patients provided written consent to participate in the study and ethical committee approval was obtained. 


\subsection{Endoscopic Radial Artery Harvest Technique}

All patients who were scheduled to undergo radial artery harvest must have met the following criteria preoperatively. First, non-dominant hand was used for the harvest. Second, Allen test was $<6$ seconds the day before surgery in the arm harvest site. Third, Pulse oximetry examination just prior to radial harvest must have been normal after occlusion of radial artery. Fourth, suitable target vessel that is $>1 \mathrm{~mm}$ with the type of stenos is mentioned previously.

Endoscopic radial artery harvesting was introduced in our institution in 2014, It was performed using the (VASOVIEW HEMOPRO 2, MAQUET Cardiovascular, Wayne; USA).

The left arm was placed on a surgical board and positioned at a $90^{\circ}$ angle. A tourniquet was placed high on the upper arm. After prepping and draping the arm, $2-3 \mathrm{~cm}$ longitudinal incision was made proximal to the wrist crease over the radial artery. A bipolar scissors (or a bisector) with low-energy electrocautery under direct vision, was used to dissect locally through the lateral muscular fascia to identify the radial artery and venae comitantes which were dissected as a pedicle. The radial artery was then clamped and pulse oximetry on the index finger was checked to assess radial and ulnar arterial flow. Heparin intravenously is then given at a dose of $2500 \mathrm{IU}$ and allowed to circulate for 2 minutes. The tourniquet was then inflated to $75-100 \mathrm{mmHg}$ over systolic blood pressure, not exceeding $250 \mathrm{mmHg}$ total.

Endoscopic harvesting of the radial artery was then performed from distal to proximal. The radial artery pedicle was then divided and ligated using the "stab and grab" technique in the antecubital fossa with an 11-blade. The distal end was ligated with a suture. After harvesting the radial artery, the forearm was then wrapped tightly with a bandage from the fingers to the elbow (i.e. distal to proximal) to evacuate the blood. All radial arteries were soaked and flushed in a solution containing a mixture of papaverine, diltiazem, saline and heparinized blood. All branches were then clipped. Patients were commenced on oral calcium channel blockers indefinitely (diltiazem) starting from postoperative day 1 or the day after extubation with the dose adjusted according to the patient heart rate.

\subsection{Histological Evaluation}

The proximal ends of radial artery were randomly collected from 8 patients and sent for histopathologic examination to evaluate the integrity of the arterial wall after endoscopic radial artery harvesting. The samples were immersed in $10 \%$ neutral buffered formalin. The length of the specimens ranged from 3 to $9 \mathrm{~mm}$ and all were processed to paraffin blocks. All paraffin embedded samples were cut at $2 \mathrm{~mm}$ by Leica RM2245 semiautomatic microtome and stained with Hematoxylin and Eosin (H\&E stain) by Tissue-Tek Prisma automatic slide stainer. The special stains Verhoef van Gieson (VVG) and Masson Trichrome (MT) were performed by ArtisanLink Pro Dako automatic slide stainer to highlight the 
elastic membranes and to assess possible fibrosis in the arteries' walls respectively.

\subsection{Radiological and Follow-Up Evaluation}

Computerized tomography (CT) coronary was performed using GE Healthcare (WI, USA). It is a multi-slice 256 detector row CT scanner. The same eight patients who had their radial artery examined histologically underwent follow-up CT coronary at 1-year interval. In addition, standard coronary angiography was also conducted in the remaining patients based on symptoms of recurrence of angina or recurrence of myocardial infarction and this was performed in 4 additional patients. All patients were followed up clinically for 1 year following surgery in the clinic and special attention to any cardiac readmission and recurrence of symptoms was made. No patient lost follow-up during the study period and the subsequent 1-year follow-up.

\subsection{Statistical Analysis}

All Data were collected prospectively in a departmental database (Patient analysis and tracking system, Dendrite clinical, UK). All data were presented as percentages, where applicable, mean and standard deviations were provided for continuous variables. Statistical analysis was performed using SPSS version 17 (SPSS, Chicago, IL, USA).

\section{Results}

\subsection{Clinical and Angiographical Data}

Fifty consecutive patients undergoing first time CABG using radial artery as a conduit were prospectively enrolled. All patients provided consent to participate in the study. As shown in Table 1, majority of patients were males with mean age of 58 years old. Most patients had hypertension, diabetes and hyperlipidemia. Mean coronary artery graft number was 3.7 per patient and the majority was isolated grafts (76\%). The target vessel for the radial artery was obtuse marginals followed by diagonals and posterior descending artery. Standard flowmeter reading after weaning from cardiopulmonary bypass using MEDISTIM (VeriQ C, elo systems, California, USA) is summarized in Table 1.

All patients were available for follow up at 1 year following the surgery. As shown in Table $2,6 \%$ of patients $(n=3)$ developed transient complication related to radial artery harvest site.

These were seen in 3 patients as follows: left thumb weakness in one patient, left wrist pain in another and left forearm hematoma in a patient who was on oral anticoagulant for atrial fibrillation. All these complications resolved within 3 months after the surgery. There was no 30-day mortality and 1-year survival was $100 \%$. During follow-up period of one year, 4 patients (8\%) required repeat revascularization by percutaneous coronary intervention (PCI) for recurrence of 
Table 1. Preoperative characteristics of study population $(n=50)$.

\begin{tabular}{|c|c|}
\hline Gender & \\
\hline Male & $44(88 \%)$ \\
\hline Female & $6(12 \%)$ \\
\hline \multicolumn{2}{|l|}{ Age (years) } \\
\hline Range & $39-77$ \\
\hline Mean \pm SD & $58.2 \pm 8.2$ \\
\hline Hypertension & $40(80 \%)$ \\
\hline Hyperlipidemia & $35(70 \%)$ \\
\hline Diabetes mellitus & $33(66 \%)$ \\
\hline \multicolumn{2}{|l|}{ Smoking status } \\
\hline Smoker & $7(14 \%)$ \\
\hline Non/ex-smoker & $43(86 \%)$ \\
\hline Previous percutaneous coronary intervention & $8(16 \%)$ \\
\hline \multicolumn{2}{|l|}{ Preoperative ejection fraction (\%) } \\
\hline Range & $30-65$ \\
\hline Mean \pm SD & $50.1 \pm 9.5$ \\
\hline \multicolumn{2}{|l|}{ Cardiac presentation } \\
\hline Angina & $22(44 \%)$ \\
\hline Non-ST elevation myocardial infarction & $22(44 \%)$ \\
\hline ST elevation myocardial infraction & $6(12 \%)$ \\
\hline \multicolumn{2}{|l|}{ Number of grafts } \\
\hline Range & $3-6$ \\
\hline Mean \pm SD & $3.7 \pm 0.7$ \\
\hline \multicolumn{2}{|l|}{ Type of anastomosis for radial artery per patient } \\
\hline Isolated & $38(76 \%)$ \\
\hline Sequential & $12(24 \%)$ \\
\hline \multicolumn{2}{|l|}{ Target vessels for radial artery per patient } \\
\hline Diagonal & $9(18 \%)$ \\
\hline Obtuse marginal & $23(46 \%)$ \\
\hline Obtuse marginal and diagonal sequential & $3(6 \%)$ \\
\hline Obtuse marginal sequential & $9(18 \%)$ \\
\hline Posterior descending/posterolateral branch of RCA & $6(12 \%)$ \\
\hline \multicolumn{2}{|l|}{ Mean flow intra-operative measurements ( $\mathrm{ml} / \mathrm{min})$} \\
\hline Range & $15-82$ \\
\hline Mean \pm SD & $41.7 \pm 17.1$ \\
\hline \multicolumn{2}{|l|}{ Not available in 6 patients } \\
\hline \multicolumn{2}{|l|}{ Pulsatility index measurement intraoperative } \\
\hline Range & $0.7-5.4$ \\
\hline Mean \pm SD & $2.4 \pm 1.0$ \\
\hline Not available in 6 patients & \\
\hline
\end{tabular}


Table 2. Postoperative outcomes and survival in the study population $(n=50)$.

\begin{tabular}{|c|c|}
\hline \multicolumn{2}{|l|}{ Complications of radial harvest at 2 weeks } \\
\hline \multicolumn{2}{|l|}{ post-op: } \\
\hline Yes & $3(6 \%)$ \\
\hline No & $47(94 \%)$ \\
\hline \multicolumn{2}{|c|}{ Complications of radial harvest at 3 months } \\
\hline \multicolumn{2}{|l|}{ post-op: } \\
\hline None & $50(100 \%)$ \\
\hline \multicolumn{2}{|l|}{ Repeat revascularization } \\
\hline Yes & $4(8 \%)$ \\
\hline No & $47(92 \%)$ \\
\hline \multicolumn{2}{|l|}{ Recurrent angina symptoms } \\
\hline Yes & $4(8 \%)$ \\
\hline No & $47(92 \%)$ \\
\hline \multicolumn{2}{|l|}{ Mortality } \\
\hline 30 days & 0 \\
\hline 1 year & 0 \\
\hline${ }^{\star}$ Patency of radial artery at 1 year & $100 \%$ \\
\hline
\end{tabular}

${ }^{*}$ Includes 8 patients who underwent CT coronary and 4 patients who underwent coronary angiography.

angina symptoms. These four patients had saphenous vein occlusion and patent radial arteries. In addition, the other 8 patients who were randomly selected underwent CT coronary angiography showed all patent radial arteries (Figure 1(a) and Figure 1(b)).

\subsection{Histological Examination}

The histopathological evaluation revealed that the walls of the harvested arteries were well preserved with normal integrity of all the layers. Most of the arteries showed thin intima with intact endothelium and internal elastic lamina (Figures 2(a)-(c)). Two of the samples displayed segments with eccentric intimal thickening (intimal cushions) and diffuse intimal thickening without deposition of lipids, calcium or inflammatory cellular reaction. This finding could be explained as a physiological response to hemodynamic changes and did not cause any luminal narrowing or any deposition of calcium or lipids. The endothelial lining was continuous.

Few focal short detachments of the endothelium were seen in some vessels, notably in the shortest specimens most likely due to the proximity to the site of vessel clamp during harvesting. The internal elastic lamina (IEL) of all cases was also continuous without tears or fragmentation of the elastic fibers (Figure 2(d) and Figure 2(e)). At the sites of intimal thickening the IEL was duplicated. Some of the samples revealed mild fibrosis of the media. The circular smooth muscle 


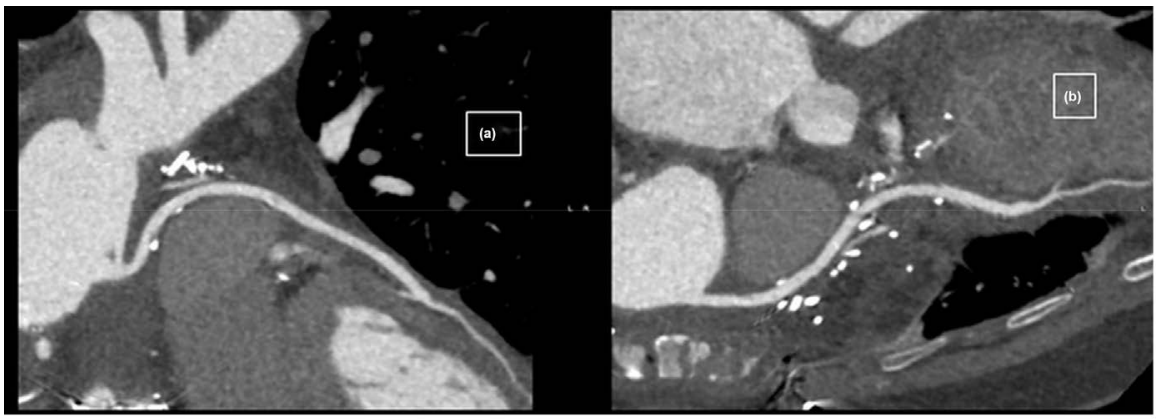

Figure 1. (a) and (b) computerized tomography (CT) coronary showing patent radial artery.

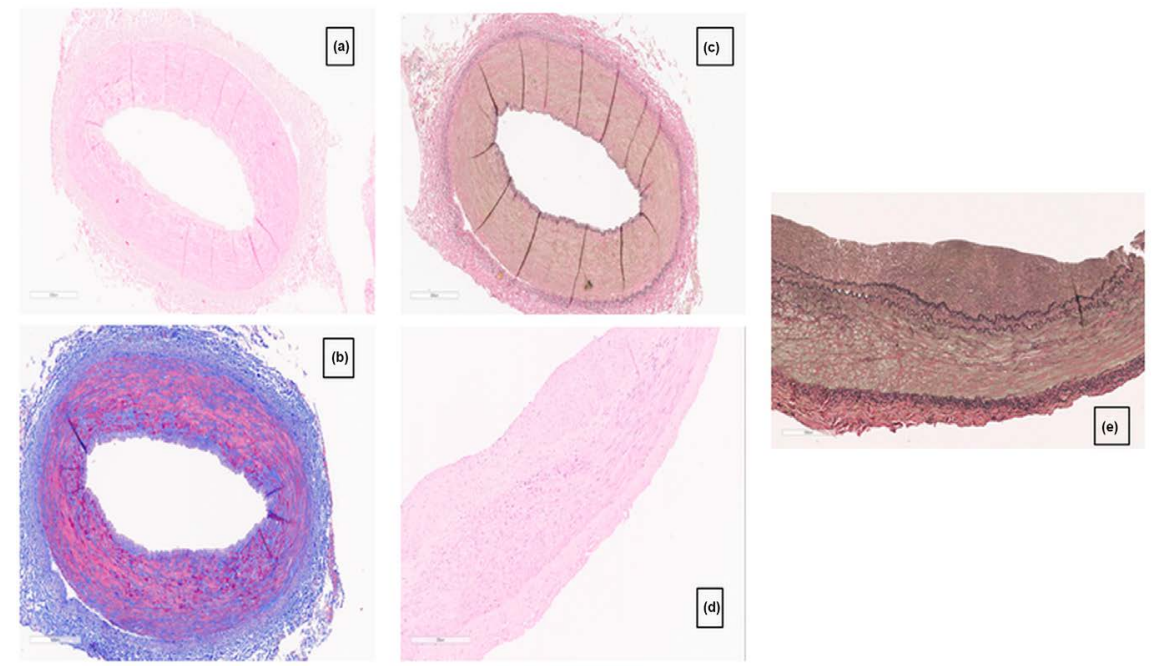

Figure 2. (a)-(e) Figures from A-C (magnification $\times 10$ ) are complete cross section of radial artery demonstrating integrity with normal architecture, patent lumen, continuous integrity of IEL and EEL. These were stained with H\&E, MT and VVG respectively. Figures $\mathrm{D} \& \mathrm{E}$ (magnification $\times 20$ ) show small intimal cushion and are stained with H\&E and VVG respectively. Abbreviations: H\&E: hematoxylin and eosin. MT: Masson Trichrome. VVG: Verhoef van Gieson. IEL: internal elastic lamina. EEL: external elastic lamina.

showed no evidence of degenerative changes, no crushing or hemorrhage. All specimens had patent lumens and were free of fresh thrombi. In conclusion, the histopathological evaluation of the segments of the harvested radial artery displayed high integrity of the wall, intact endothelial lining, patent lumens and no significant pathological abnormalities. None of the arteries showed traumatic injury that could be related to the surgical procedure.

\section{Discussion}

Radial artery has gained a wide popularity as a second arterial conduit in coronary artery bypass graft surgery (CABG) owing to its characteristics and lower morbidity rates associated with its harvest. However, the overall use of radial artery is still underutilized with a report of the society of society of thoracic surgeons in 2008 showing that $6 \%$ of patients received a radial artery as a con- 
duit [7]. Endoscopic radial artery harvesting has been shown to have similar patency to open radial artery harvests up to 3 years with a lower rate of post-operative wound infection, hematoma formation, neurological dysfunction, improved cosmetic appearance and less pain [8] [9]. However, because the endoscopic technique is conducted in a closed small space, there are concerns raised regarding the potential of thermal and mechanical injury to the endothelium of the radial artery conduit [9]. In a prospective randomized trial, Shapira et al. have assessed the effect of endoscopic radial artery harvest technique on the vasoreactivity and the structural integrity of the radial artery by comparing to the open conventional technique. The author demonstrated that structural integrity and vasoreactivity of the endoscopically harvested radial artery remained intact [10].

Histologically, the radial artery has a thin continuous intima of endothelial cells, a single internal elastic lamina and a relatively thick media of packed smooth muscle cells which predisposes to spasm [8]. In addition, the radial artery conduit is often free from atherosclerosis and has metabolically active endothelial and smooth muscle layers which contribute to greater flow velocity and the ability to remodel to target vessel size (9). These findings are well in line with our findings histologically. In addition, in the samples we examined histologically, the use of endoscopic technique did not result in any demonstrable thermal or traumatic injury to the harvested vessel. This demonstrates the safe use of endoscopic technique in radial artery harvest. In addition, our excellent rates of patency of the radial artery used at 1 year follow up with few post-operative morbidities show promising results in endoscopically harvested radial arteries and their use as a second arterial conduit in CABG.

\section{Limitations of the Study}

There are few limitations in our study. First, our sample size of 50 patients is small. Secondly, we used random 8 cases to examine them both histologically and by radiological evaluation. This may have skewed our results. However, these cases were randomly chosen, and we still believe that these results will be reproduced in the remaining cases if they have been studied histologically. Third, our follow up is generally short at 1 year. Fourth, we only studied the use of endoscopic harvest technique on histological appearance of the radial artery and we did not compare that to open harvest technique.

\section{Conclusion}

In this study, the use of endoscopic radial artery harvest was associated with few short-term morbidities which are self-limiting and excellent patency rates. In addition, the use of endoscopic technique in radial artery harvest showed no evidence of any thermal or traumatic injuries to the radial arteries when examined histologically. The use of endoscopic radial artery appears safe and the results are excellent. 


\section{Conflicts of Interest}

The authors declare no conflicts of interest regarding the publication of this paper.

\section{References}

[1] Cao, C., Ang, S.C., Wolak, K., Peeceeyen, S., Bannon, P. and Yan, T.D. (2013) A Meta-Analysis of Randomized Controlled Trials on Mid-Term Angiographic Outcomes for Radial Artery versus Saphenous Vein in Coronary Artery Bypass Graft Surgery. Annals of Cardiothoracic Surgery, 2, 401-407.

[2] Deb, S., Cohen, E.A., Singh, S.K., Une, D., Laupacis, A., Fremes, S.E., et al. (2012) Radial Artery and Saphenous Vein Patency More than 5 Years after Coronary Artery Bypass Surgery: Results from RAPS (Radial Artery Patency Study). Journal of the American College of Cardiology, 60, 28-35.

https://doi.org/10.1016/j.jacc.2012.03.037

[3] Navia, J.L., Olivares, G., Ehasz, P., Gillinov, A.M., Svensson, L.G., Brozzi, N., et al. (2013) Endoscopic Radial Artery Harvesting Procedure for Coronary Artery Bypass Grafting. Annals of Cardiothoracic Surgery, 2, 557-564.

[4] Patel, A.N., Henry, A.C., Hunnicutt, C., Cockerham, C.A., Willey, B. and Urschel Jr., H.C. (2004) Endoscopic Radial Artery Harvesting Is Better than the Open Technique. Annals of Thoracic Surgery, 78, 149-153. https://doi.org/10.1016/j.athoracsur.2004.03.001

[5] Wu, H.B., Hu, R., Wang, Z.W., Hu, Z.P., Li, L.C., Wu, Z.Y., et al. (2014) Endoscopic Radial Artery Harvesting Does Not Compromise Graft Patency for Coronary Artery Bypass Graft: A Meta-Analysis of 2782 Patients. Heart, Lung and Circulation, 23, 1084-1090. https://doi.org/10.1016/j.hlc.2014.03.023

[6] Cao, C., Tian, D.H., Ang, S.C., Peeceeyen, S., Allan, J., Fu, B. and Yan, T.D. (2014) A meta-analysis of endoscopic versus conventional open radial artery harvesting for coronary artery bypass graft surgery. Innovations (Phila), 9, 269-275.

[7] Tabata, M., Grab, J.D., Khalpey, Z., Edwards, F.H., O’Brien, S.M., Cohn, L.H., et al. (2009) Prevalence and Variability of Internal Mammary Artery Graft Use in Contemporary Multivessel Coronary Artery Bypass Graft Surgery: Analysis of the Society of Thoracic Surgeons National Cardiac Database. Circulation, 120, 935-940. https://doi.org/10.1161/CIRCULATIONAHA.108.832444

[8] Rehman, S.M., Yi, G. and Taggart, D.P. (2013) The Radial Artery: Current Concepts on Its Use in Coronary Artery Revascularization. Annals of Thoracic Surgery, 96, 1900-1909. https://doi.org/10.1016/j.athoracsur.2013.06.083

[9] Dimitrova, K.R., Dincheva, G.R., Hoffman, D.M., DeCastro, H., Geller, C.M. and Tranbaugh, R.F. (2013) Results of Endoscopic Radial Artery Harvesting in $1577 \mathrm{~Pa}$ tients. Innovations (Phila), 8, 398-402.

[10] Shapira, O.M., Eskenazi, B.R., Anter, E., Joseph, L., Christensen, T.G., Hunter, C.T., et al. (2006) Endoscopic versus conventional Radial Artery Harvest for Coronary Artery Bypass Grafting: Functional and Histologic Assessment of the Conduit. Journal of Thoracic and Cardiovascular Surgery, 131, 388-394.

https://doi.org/10.1016/j.jtcvs.2005.07.036 